\title{
Propuesta didáctica para analizar los enunciados de los problemas de matemáticas
}

Israel Mazario Triana

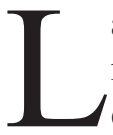

a resolución de problemas es uno de los aspectos más importantes de la enseñanza de las matemáticas. Al respecto, se argumenta que es una actividad cognoscitiva a través de la cual se pone en práctica el principio general de aprendizaje activo propugnado por la escuela contemporánea, posibilitando, además, enfatizar en los procesos de pensamiento y en la aplicación de los conocimientos en diversas situaciones de las ciencias y la vida cotidiana.

A pesar de este reconocimiento, es significativo el índice de fracaso de los estudiantes en la resolución de problemas, razón por la cual, esta problemática se ha constituido en un campo de creciente interés para la investigación educativa.

En esta dirección, cuando se presentan a los estudiantes los problemas de matemáticas, la primera dificultad que se constata es la relacionada con el análisis de los enunciados, lo que también constituye un obstáculo para avanzar hacia la solución correcta del problema.

Ante esto, se requiere de una intervención didáctica adecuada para que los estudiantes puedan resolver los problemas planteados en las clases, de tal manera que se promueva su buen desempeño escolar.

En este texto se tiene por objetivo elaborar una propuesta didáctica que oriente a los estudiantes en el procedimiento para analizar los enunciados de los problemas de matemáticas.

\section{Analizar el enunciado de un problema de matemáticas}

Como se ha dicho ya, esta acción constituye la dificultad inicial que se presenta al resolver un problema, se manifiesta desde el momento en que el estudiante enfrenta el problema y trata de descomponerlo en sus partes con el objetivo de identificar los datos que le aporta el enunciado, las relaciones establecidas entre los diferentes componentes de la situación planteada y, simultáneamente, determinar las interrogantes que debe responder; sin embargo, esta actividad analítica se complementa con otra de síntesis en la cual se logra una restructuración consciente de la situación que se desea resolver. De conformidad con esta acepción, Miriam Heller (1998: 84) considera que a través de la síntesis: "el individuo estructura situaciones por sí mismo, va más allá de la información que le brinda el medio organizándola e integrándola con sus necesidades e intereses". Estas características, según expresan Vicente Pérez et al. (2005), no son exclusivas del pensamiento matemático, sino que se hacen presentes en otras formas de pensar.

Ahora bien, cuando a los estudiantes se les presentan problemas, el lenguaje es utilizado como un medio para transmitir las instrucciones que preceden el objetivo a lograr, esta información es dada en forma verbal o escrita, pero es usual combinarla o reforzarla con la incorporación del recurso visual (gráficas, tablas, diagramas, etc.), que contribuye a que los estudiantes ganen claridad en la comprensión del problema. Estos recursos pueden 
tiene que atender la organización definitiva de las preguntas y recomendaciones, además, generar la participación activa de los estudiantes a través del propio proceso de resolución de los problemas. Este medio queda en definitiva elaborado al transcurrir varias sesiones de trabajo con los problemas, en tanto se revelan precisamente las operaciones esenciales para resolverlos gradualmente. De este modo, una vez que se llegan a determinar un conjunto de interrogantes y sugerencias interrelacionadas, éstas se constituyen en prescripciones que especifican el proceso de resolución de problemas, y posteriormente se registra el listado en papel. Los estudiantes trabajan durante un tiempo razonable con esta guía didáctica, hasta que puedan prescindir de este medio.

Obviamente, la efectividad de este recurso presupone que los alumnos se familiaricen paulatinamente con sus instrucciones y las interioricen, para que posteriormente, al aplicarlas, realicen los ajustes en relación con el tipo de problema a solucionar. En efecto, las preguntas y sugerencias que contiene la guía didáctica, como se ilustra a continuación, no pueden ser incorporadas en su totalidad a todos los problemas, ni agotan todas las que puedan surgir.

\section{Guía didáctica del estudiante para analizar el enunciado de problemas de matemáticas}

Después de la lectura cuidadosa del enunciado, debes preguntarte:

1. ¿Cuáles son los elementos del problema que más te han llamado la atención?

2. ¿Has comprendido todas las palabras del enunciado del problema?

3. ¿Lo puedes relacionar con algún concepto, disciplina, experiencia, situación o problema anterior?

4. ¿Puedes expresar de qué trata el problema?

5. ¿Debes repetir la lectura del enunciado del problema para comprenderlo? ¿Puedes precisar los elementos del mismo que te generan dificultad en su comprensión?

6. ¿Qué se pide hallar?, o ¿ya conoces la demanda del problema?
7. ¿Qué datos puedes extraer del problema?

8. ¿Consideras que los datos del problema son suficientes para resolverlo?, ¿están de acuerdo con los que has manejado en alguna experiencia previa?

9. ¿Existe alguna relación entre estos datos?

10. ¿Puedes representar estos datos o la situación que se te presenta a través de un gráfico, tabla, etc., que te ayude a resolverlo?

11. ¿Consideras que necesitas para resolver el problema algún dato que no aparece en el mismo?

12. ¿Qué conocimientos matemáticos o de otras disciplinas consideras convenientes para resolver el problema?

13. ¿Conoces algún algoritmo o estrategia para resolver el problema?

14. Por último, piensa de otra manera o escribe de otra forma el problema, para que se facilite su comprensión.

También, en las clases se analizan los enunciados de los problemas en grupo, y en caso de ser necesario se redactan de otra manera, lo que resulta muy eficaz para la elaboración de nuevos enunciados o al estructurar un enunciado a partir de los datos, lo que simultáneamente dio la posibilidad al docente de reflexionar sobre "su enunciado".

La aplicación de la propuesta didáctica, a diferencia de la forma tradicional de analizar los problemas de matemáticas, se enmarca en un contexto de enseñanza definido por un conjunto de actividades que se seleccionan por su potencial educativo, de manera que permita a los estudiantes generar reflexión y juzgar sus propias formas de actuar.

\section{Fuentes de consulta}

Delgado, K. (2011). Aprendizaje colaborativo. Teoría y práctica. Perú: Editorial San Marcos.

Heller, M. (1998). El arte de enseñar con todo el cerebro. Venezuela: Fotoprin C.A.

Pérez, V. et al. (2005). Procesos psicológicos básicos. Un análisis funcional. México: Pearson. Prentice Hall. 